\title{
Designs of Two-Element Optical Refracting System to Achieve Uniform Laser Beam Profile
}

\author{
Abdallah K. Cherri*, Nabil I. Khachab, Mahmoud K. Habib \\ Electrical Engineering Department, College of Engineering and Petroleum, Kuwait University, Safat, Kuwait \\ Email: *abdallah.cherri@ku.edu.kw
}

How to cite this paper: Cherri, A.K., Khachab, N.I. and Habib, M.K. (2017) Designs of Two-Element Optical Refracting System to Achieve Uniform Laser Beam Profile. Journal of Applied Mathematics and Physics, 5, 2371-2385.

https://doi.org/10.4236/jamp.2017.512194

Received: October 6, 2017

Accepted: December 25, 2017

Published: December 28, 2017

Copyright $\odot 2017$ by authors and Scientific Research Publishing Inc. This work is licensed under the Creative Commons Attribution International License (CC BY 4.0).

http://creativecommons.org/licenses/by/4.0/

\begin{abstract}
Various specific laser irradiance distribution outputs are needed in many applications. To fulfill this need, a detailed step-by-step design procedure for split refracting system is proposed for three types of laser beams transformation: 1) Annular-uniform-to-uniform; 2) Annular-Gaussian-to-uniform; and 3) Gaussian-to-uniform to obtain the required laser irradiance distributions. Mathematical expressions of the two Plano-aspheric surfaces are derived for each type. The proposed designs take into account few important parameters such as the system length, the surfaces radii of curvature, the annular beam starting cone angle, and the beams power ratio. Further, the proposed designs are much better than the ones, which were previously reported.
\end{abstract}

\section{Keywords}

Annular Laser Beam, Refracting System, Lens Design

\section{Introduction}

The TEM00 laser type produces output beam with either Gaussian or near-Gaussian intensity profiles [1] [2]. It is one of the majorities of laser types in current use for many applications in which the laser beam is being focused to a small spot. However, there are many other applications where uniform intensity distributions are needed. For example, in coherent image processing, pattern recognition, Fourier transforms based correlation, and materials processing tasks, a uniform intensity distribution is required to illuminate evenly the entire processed area. In addition, uniform illumination benefits a wide range of other applications such as in machine vision, industrial inspection, microphotolithography, and in medicine [3] [4] [5] [6] [7]. Thus, the conversion of Gaussian and non-Gaussian laser beams to uniform beam profiles appears to be very beneficial to many applications. Refractive optical system method is among many successful 
and efficient methods that can make this conversion. The method relies on geometric optics for designing laser beam shaping systems [8]-[19]. Over the years, one-element and two-element refracting systems have been proposed to achieve beam shaping where Gaussian and annular-Gaussian beam profiles were transformed to uniform beams, as well as to Bessel beam profiles [20]-[25].

In this paper, two-element (instead of one-element) refracting beam shaping systems are designed. Two separate lenses (input and output) with aspheric surfaces of revolution are designed to convert: 1) annular-uniform beam profile to uniform profiles; 2) annular-Gaussian beam to uniform profiles; and 3) Gaussian beam to uniform beam profiles. Detailed step-by-step design is outlined to derive the mathematical expressions that represent the curvature and the asphericity of the input and output lenses. In addition, discussions of the various parameters that are affecting the proposed design are presented such the overall length of the system, the radii of curvature of the surfaces, and the power ratio.

\section{Design Procedure}

The two-element design refracting optical system for beam shaping is governed by two main conditions that are derived from the geometrical optics of ray tracing, namely:

1) The input rays that enter the first lens and leave the second lens must have the same optical path length.

2) The input rays to the first lens and the corresponding output rays from the second lens must be parallel to each other.

In addition, a third condition which is related to the beams profiles that are being converted is imposed by the energy conservation, i.e.:

3) The ratio of the input beam power (through the first lens) to the output beam power (from the second lens) must equal to a constant.

The geometrical configuration of the two-lens refracting system is shown in Figure 1 where the incident beam has annular-uniform profile while the exit beam will have uniform profile. Consequently, there is no radiation passing through the central aperture of the input lens. The two aspheric lenses (made from glass with index of refraction $n=1.5172$ ) are separated by air (index of refraction $n=1$ ) by a distance $D . \theta_{i i}$ and $\theta_{r i}$ are the incident and refracted angles of the rays that enter and exit the first aspheric lens while $\theta_{r o}$ and $\theta_{i o}$ are the incident and refracted angles of the rays that reached the second aspheric lens. It is worth mentioning that the surface of the input convex aspeheric lens is designed to map the rays that enter the first half section of convex aspheric lens to the half section of the second aspheric lens after traveling in air. Further, the surface of the output concave aspeheric lens is designed to receive the rays reaching the lens and refract them so that they exit parallel to the first incident rays. The two designed surfaces will be designated mathematically as $y_{i}\left(r_{i}\right)$ and $y_{o}\left(r_{o}\right)$ as functions of the radial distances $r_{i}$ and $r_{o}$ of the input and output lenses, respectively.

From the geometry of the set-up in Figure 1, one can deduce that: 


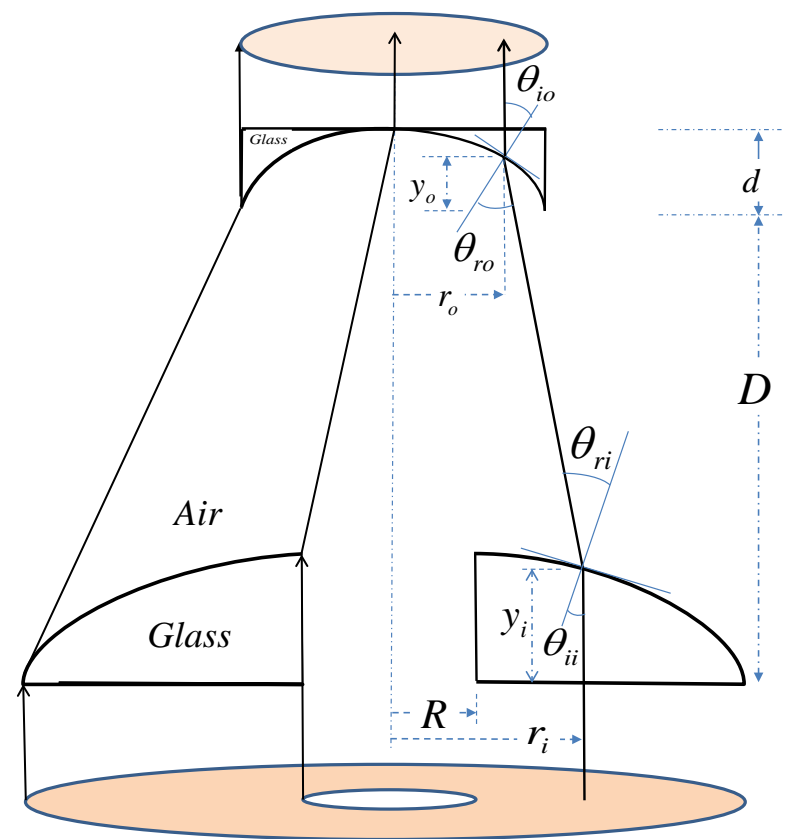

Figure 1. The geometric set-up of the two-lens refracting system beam transformation.

$$
\tan \left(\theta_{r i}-\theta_{i i}\right)=\tan \left(\theta_{r o}-\theta_{i o}\right)=\frac{r_{i}-r_{o}}{D-y_{i}+y_{o}}
$$

Next, the above first condition is translated into the following equation:

$$
n y_{i}+\sqrt{\left(r_{i}-r_{o}\right)^{2}+\left(D-y_{i}+y_{o}\right)^{2}}+n\left(d-y_{o}\right)=f
$$

where $f$ is a constant. Adding $(-n D)$ term to both sides of Equation (2), yields:

$$
-n D+n y_{i}-n y_{o}+\sqrt{\left(r_{i}-r_{o}\right)^{2}+\left(D-y_{i}+y_{o}\right)^{2}}=f-n d-n D
$$

Let $f^{\prime}=f-n d-n D$ and use Equation (1), we obtain:

$$
\frac{-n}{\tan \left(\theta_{r i}-\theta_{i i}\right)}+\frac{\sec \left(\theta_{r i}-\theta_{i i}\right)}{\tan \left(\theta_{r i}-\theta_{i i}\right)}=\frac{f^{\prime}}{r_{i}-r_{o}}
$$

Further, the second condition of input and output ray parallelism dictates that both input and output surfaces slopes must be equal, namely:

$$
\frac{\mathrm{d} y_{i}}{\mathrm{~d} r_{i}}=\frac{\mathrm{d} y_{o}}{\mathrm{~d} r_{o}}=-\tan \theta_{i i}=-\tan \theta_{i o}
$$

Furthermore, by applying Snell's law $\left(n \sin \theta_{i i}=\sin \theta_{r i}\right)$ at the input lens and using trigonometric identities, one can obtain the following important relationship from Equation (3) and Equation (4):

$$
\frac{\mathrm{d} y_{i}}{\mathrm{~d} r_{i}}=\frac{\mathrm{d} y_{o}}{\mathrm{~d} r_{o}}=\frac{-1}{\sqrt{\left(\frac{f^{\prime}}{r_{i}-r_{o}}\right)^{2}+n^{2}-1}}
$$

Equation (5) is the fundamental equation that will be used to achieve the main 
objective of the two-element design, which is to obtain the mathematical expressions for the input and the output surfaces of the lenses $y_{i}\left(r_{i}\right)$ and $y_{o}\left(r_{o}\right)$. The following is a step-by-step design procedure to achieve this objective:

1) Specify the type of glass used for lenses: obtain the index of refraction $n$.

2) Specify the length of the system by selecting the constant $f$.

3) Specify the power transfer ratio $k$ between the input and the output beams.

4) Obtain the relationship between the radial distances $r_{i}$ and $r_{o}$ from the power ratio $k$.

5) Obtain the numerical values for the surfaces' slopes $\mathrm{d} y_{i} / \mathrm{d} r_{i}$ and $\mathrm{d} y_{o} / \mathrm{d} r_{o}$ from Equation (5).

6) Obtain the numerical values of the surfaces $y_{i}\left(r_{i}\right)$ and $y_{o}\left(r_{o}\right)$ by numerical integration.

7) Apply appropriate initial values on the surfaces $y_{i}\left(r_{i}\right)$ and $y_{o}\left(r_{o}\right)$ to obtain the constants of integrations.

Note that the least-squared polynomial curve-fitting routine will be used to find the mathematical functions of the surfaces $y_{i}\left(r_{i}\right)$ and $y_{o}\left(r_{o}\right)$. Moreover, in the above design procedure one needs to consider other important parameters such as:

1) The overall system length, which is preferred to be as small as possible to be practical and to account for power absorption.

2) A small value for the starting surface slope $\mathrm{d} y / \mathrm{d} r$ is desirable to provide for less diffraction and to make the fabrication of the lens much easier. Further the starting cone angle is critical for annular beams.

3) Large radii of curvature $\rho(r)$ for the surfaces are preferable to help managing the fabrication process. $\rho(r)$ of any surface is given by:

$$
\rho(r)=\frac{\left[1+(\mathrm{d} y / \mathrm{d} r)^{2}\right]^{3 / 2}}{\mathrm{~d}^{2} y / \mathrm{d} r^{2}}
$$

Consequently, the second derivative of the starting surface slope $d^{2} y / d r^{2}$ needs to be small too.

\section{Laser Beam Transformation}

In this section, the above-mentioned design procedure will be applied to transform three different types of beams and they are compared to three previously reported one-element refracting systems [20] [21].

\subsection{Annular-Uniform to Uniform Beam Transformation}

As shown in Figure 1, uniform input annular beam irradiance will be redistributed by the output lens to form a circular uniform output beam. The input surface is designed in such a way to refract the beam forward and inward onto the second surface. The second surface is designed to reorient the refracted beam upward and parallel to the original beam. The energy balance condition implies that we must define a constant ratio of intensities between the input and the 
output beams. These intensities, in turn, define the respective cross-sectional areas of the input and the output surfaces. Thus, a ratio $k$, between the cross-sectional areas can be set as:

$$
\frac{\pi\left(r_{i}^{2}-R^{2}\right)}{\pi r_{o}^{2}}=k^{2}
$$

where $r_{i}$ and $R$ are the outer and the inner radii of the annular beam, and $r_{o}$ is the radius of the output circular uniform beam. Equation (7) is solved to obtain:

$$
\begin{aligned}
& r_{i}=\sqrt{k^{2} r_{o}^{2}+R^{2}} \\
& r_{o}=\sqrt{\left(r_{i}^{2}-R^{2}\right) / k^{2}}
\end{aligned}
$$

Equations (7) and (8) declare that the infinitesimal annular area at each input radial position $\left(r_{i}\right)$ and the infinitesimal annular area at each corresponding output radial position $\left(r_{o}\right)$ form a constant ratio $k$. In other word, this means that the value of $k$ is the same at each radial position $\left(r_{p} r_{o}\right)$. Equation (8) will be used along with Equation (5) to develop various designs for the two-lens refracting system transformation as detailed below. In addition, the proposed designs will demonstrate the effects of changing the inner radius $R$ and the power ratio $k$ on the design parameters. The first proposed design will utilize the same parameters of Reference [19], namely $R=10 \mathrm{~cm}$ and $k=2$, for comparison.

The first step in the design is to choose a reasonable value of the constant $f^{\prime}$, which leads to selecting a reasonable value for the length $D$. Note that the starting value of the surface slope, the initial value of the radius of curvature, and the value of $D$ are related to each other's. These initial values are plotted in Figure 2(a) as function of $f^{\prime}$. The following algorithm provides the steps needed to obtain the curves in Figure 2(a):

1) Set $r_{o}=0 ; r_{i}=R=10 \mathrm{~cm} ; y_{i}=y_{o}=0$; and for each value of $f^{\prime}$ from 1 to 9 , do the following:

2) Calculate $\mathrm{d} y_{i} / \mathrm{d} r_{i}$ from Equation (5)

3) Calculate $\theta_{i i}$ from Equation (4)

4) Calculate $\theta_{r i}$ from Snell's law $n \sin \theta_{i i}=\sin \theta_{r i}$

5) Calculate $D$ from Equation (1)

6) Calculate $\rho(r)$ from Equation (6)

From Figure 2(a), a value $f^{\prime}=5.75$, which corresponds to $D=16.54 \mathrm{~cm}$ is selected as the closest value to $D=16.59 \mathrm{~cm}$ used in Reference [19]. Using this selected value of $f^{\prime}$ in Equation (5) along with Equation (8), we obtain the numerical values for the surface slopes by using polynomial curve-fitting that approximate the equations for $\mathrm{d} y / \mathrm{d} r$ as:

$$
\begin{aligned}
& \frac{\mathrm{d} y_{i}}{\mathrm{~d} r_{i}}=-(0.0019) r_{i}^{4}+(0.0915) r_{i}^{3}-(1.6273) r_{i}^{2}+(12.8542) r_{i}-38.82 \\
& \frac{\mathrm{d} y_{o}}{\mathrm{~d} r_{o}}=\left(7.4819 \times 10^{-5}\right) r_{o}^{4}-\left(6.1302 \times 10^{-4}\right) r_{o}^{3}-(0.0014) r_{o}^{2}+(0.0159) r_{o}-0.7827
\end{aligned}
$$




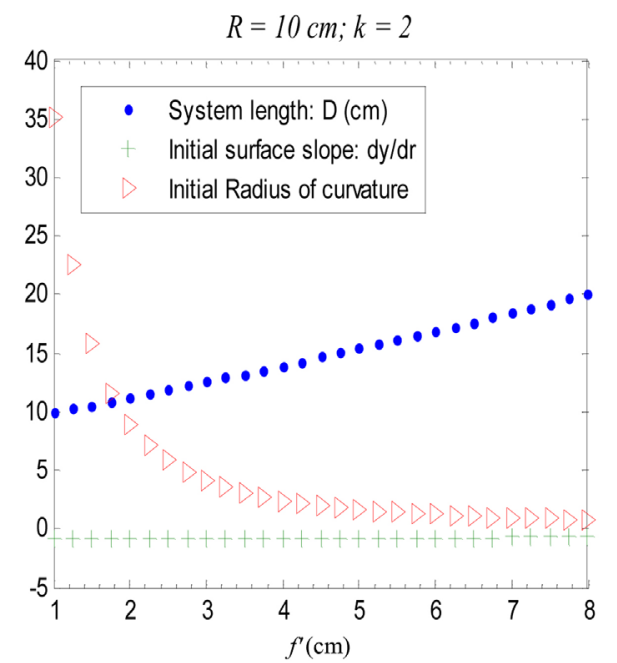

(a)

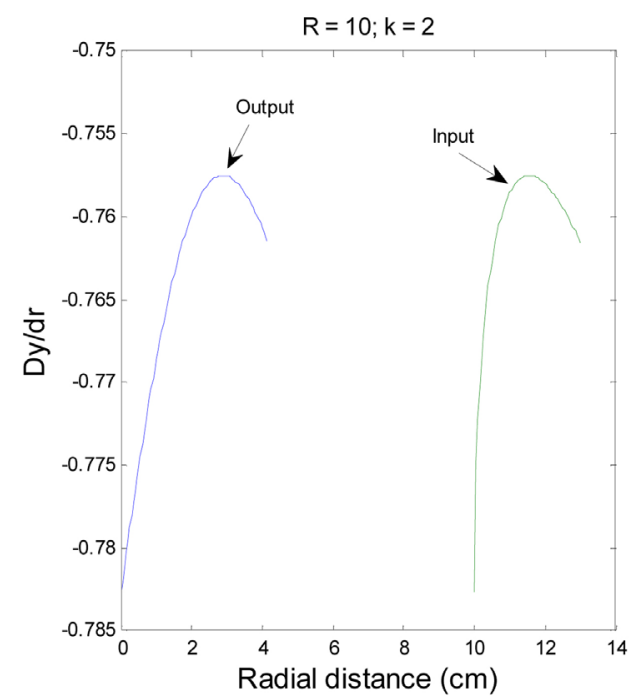

(b)

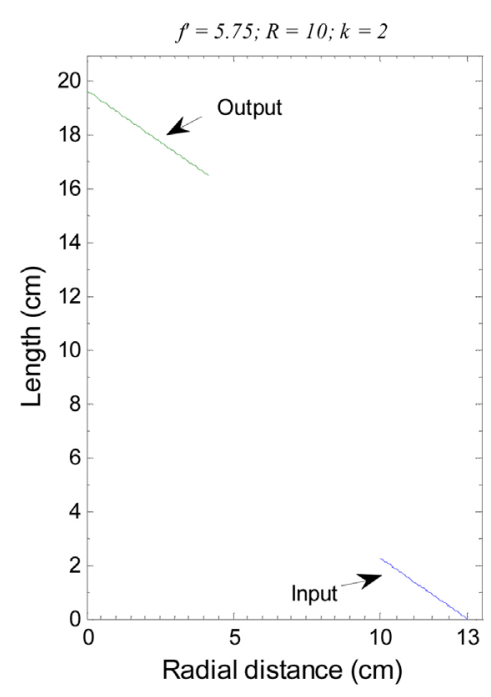

(c)

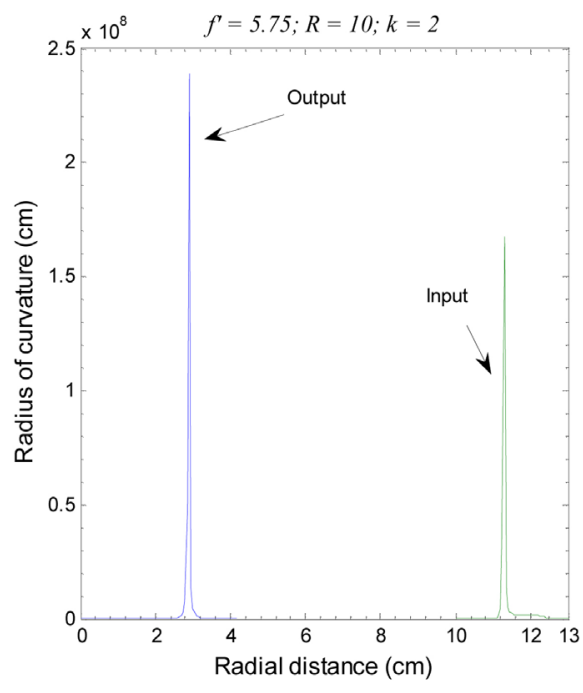

(d)

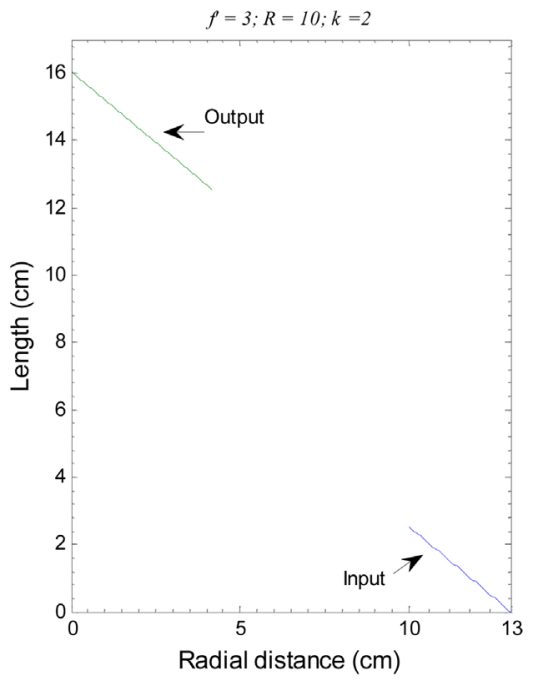

(e)

Figure 2. Two-element refracting system design for $R=10 \mathrm{~cm}, k=2, D=16.54 \mathrm{~cm}$ : (a) selecting $f$; (b) surface slopes $\mathrm{d} y_{i} / \mathrm{d} r_{i}$ and $\mathrm{d} y_{o} / \mathrm{d} r_{o}$; (c) the two surfaces of the lenses; (d) input and output radii of curvatures; (e) the two surfaces of the lenses for shorter system length when $f^{\prime}=3$.

Figure 2(b) shows the surfaces slopes for this design. Now, by integrating Equation (9) and using the initial conditions $y_{i}\left(r_{i}=13\right)=0$ and $y_{o}\left(r_{o}=0\right)=3.17$ :

$$
\begin{aligned}
y_{i}\left(r_{i}\right)= & -\left(3.8611 \times 10^{-4}\right) r_{i}^{5}+(0.0229) r_{i}^{4}-(0.5424) r_{i}^{3} \\
& +(6.4271) r_{i}^{2}-(38.82) r_{i}+99.9797 \\
y_{o}\left(r_{o}\right)= & \left(1.4964 \times 10^{-5}\right) r_{o}^{5}-\left(1.5325 \times 10^{-4}\right) r_{o}^{4}-\left(4.5245 \times 10^{-4}\right) r_{o}^{3} \\
& +(0.008) r_{o}^{2}-(0.7827) r_{o}+3.1726
\end{aligned}
$$

Figure 2(c) illustrates the final surfaces of the lenses. Large radii of curvatures for the surfaces are noticed as shown in Figure 2(d). For the input surface, the radius of curvature starts from a value of $921.5 \mathrm{~cm}$ reaching a maximum of $1.673 \times$ $10^{8} \mathrm{~cm}$; then it goes to a value of $13,840 \mathrm{~cm}$; while for the output surface, the 
radius of curvature starts from a minimum value of $8050 \mathrm{~cm}$ reaching a maximum value of $2.923 \times 10^{8} \mathrm{~cm}$ then falls to a minimum value of $6333 \mathrm{~cm}$. With respect to the overall system length of the proposed design, it is found to be $19.69 \mathrm{~cm}$ compared to $24.08 \mathrm{~cm}$ in Reference [20]; whereas the starting cone angle $\tan ^{-1}\left(D-y_{i}+y_{o}\right) / R$, is calculated to be $59.27^{\circ}$ compared to $58.92^{\circ}$ in Reference [19].

The previous design was introduced for comparison purposes. However, a better and shorter system length can be easily found when selecting a lower value for $f$. For instance, a design for $f^{\prime}=3 \mathrm{~cm}$, which correspond to $D=12.56 \mathrm{~cm}$, is illustrated in Figure 2(e) for which the overall length of the system design is reduced to $16.05 \mathrm{~cm}$ compared to $24.08 \mathrm{~cm}$ in Reference [19], with a starting cone angle equal to $53.53^{\circ}$ compared to $58.92^{\circ}$. Next, the effects on the design parameters when the power ratio is changed to $k=4,\left(f^{\prime}=5.75, R=10 \mathrm{~cm}\right)$ is shown in Figure 3(a). It is observed that the output beam radius $r_{o}$, the overall system length, and the starting cone angle decrease, respectively, to $2.1 \mathrm{~cm}$ (was 4.15 ), $18.15 \mathrm{~cm}$ (was 24.08 ), and $57.66^{\circ}$ (was $58.92^{\circ}$ ). On the other hand, when the power ratio is set to a value $k=0.707$ (lower than 1), as illustrated in Figure 3(b), then the opposite happened, i.e., the circular beam radius $r_{o}$, the overall system length, and the starting cone angle increase to $11.76 \mathrm{~cm}, 23.26 \mathrm{~cm}$, and $65.34^{\circ}$, respectively. Furthermore, the importance of the starting cone angle from the inner radius $R$ of an annular beam is discussed. This issue will affect the overall length of the two-element lens design for a fixed power ratio $k$, in addition to other factors such as the initial surface slope and the initial radius of

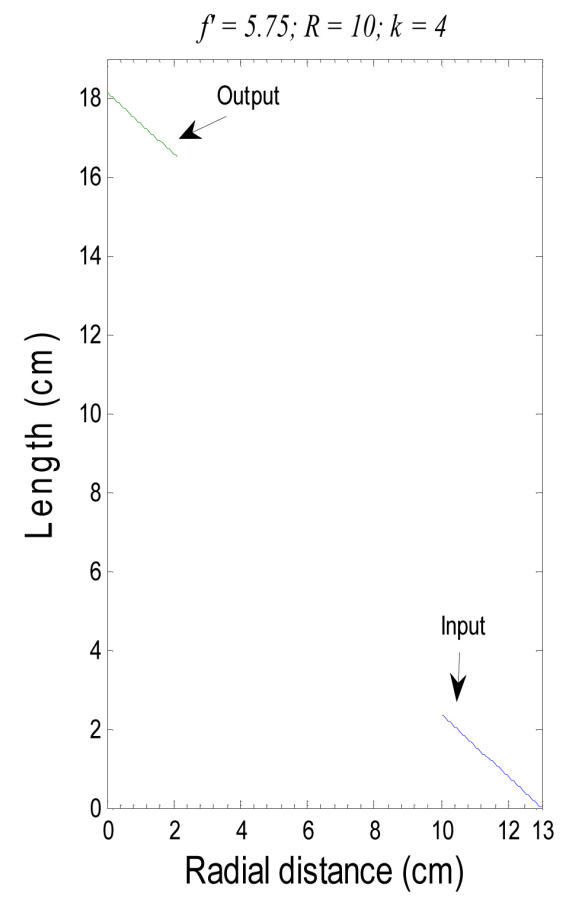

(a)

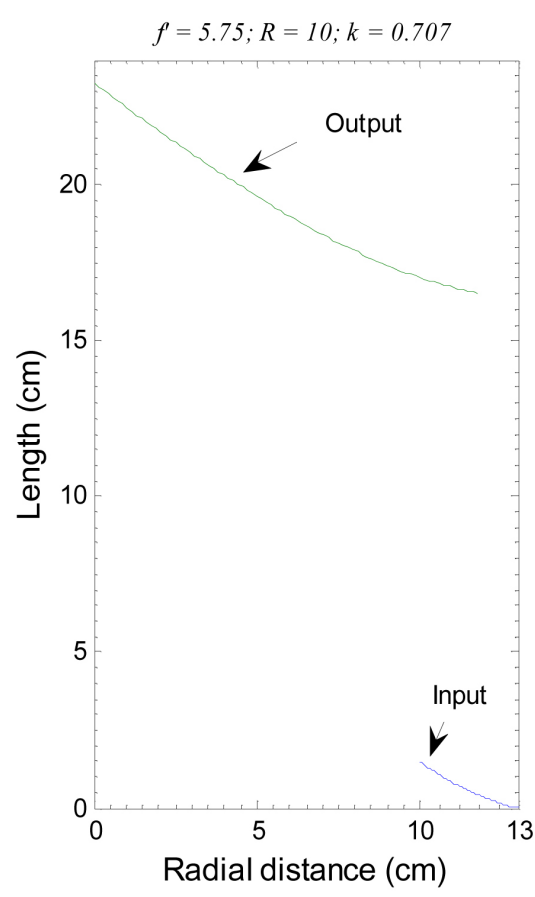

(b)

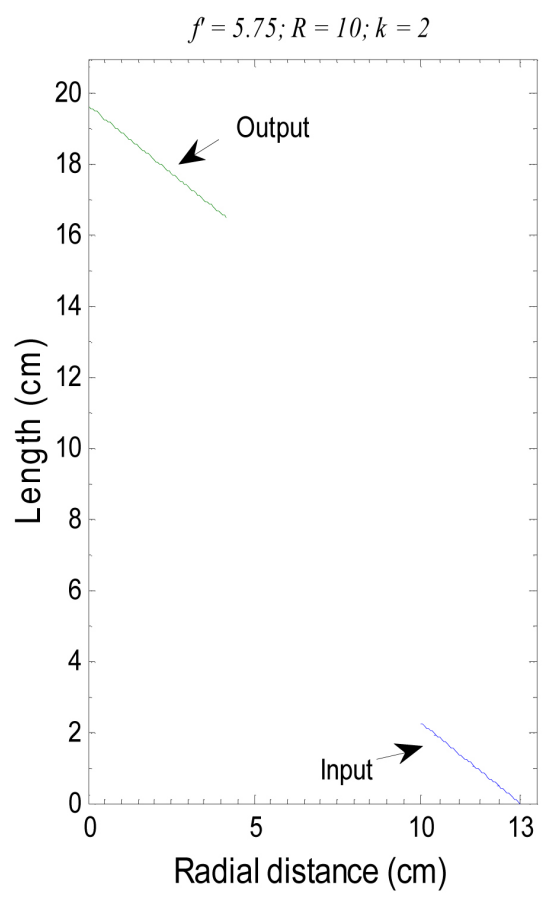

(c)

Figure 3. Effects of the power ratio on the designed surfaces for $f^{\prime}=5.75 \mathrm{~cm}, R=10 \mathrm{~cm}$ : (a) $k=4$; (b) $k=0.707$; and (c) $k=2$. 


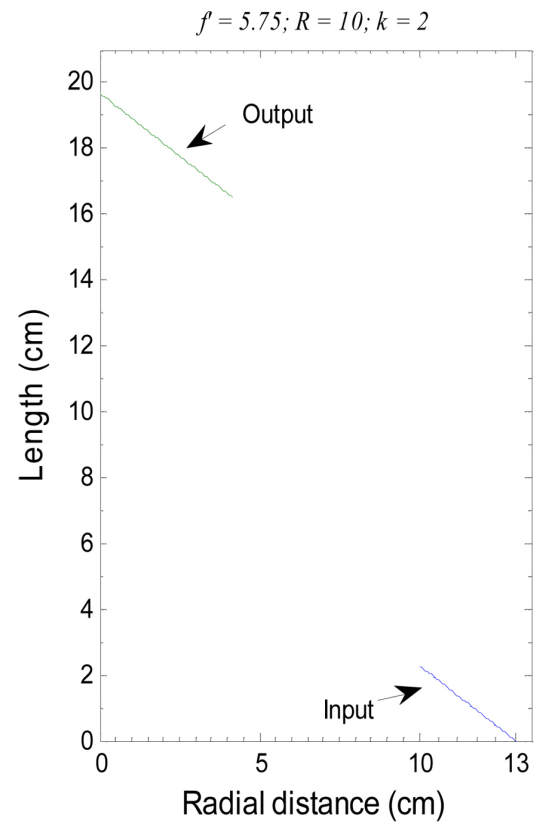

(a)

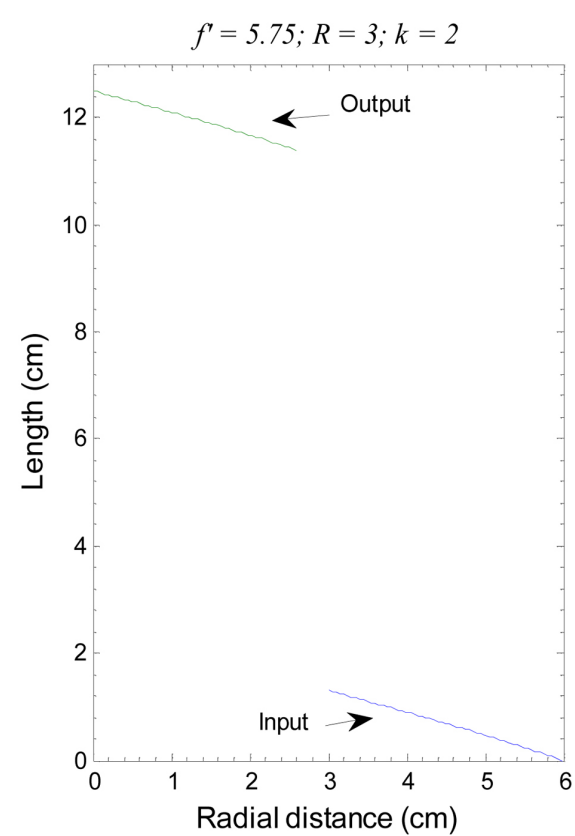

(b)

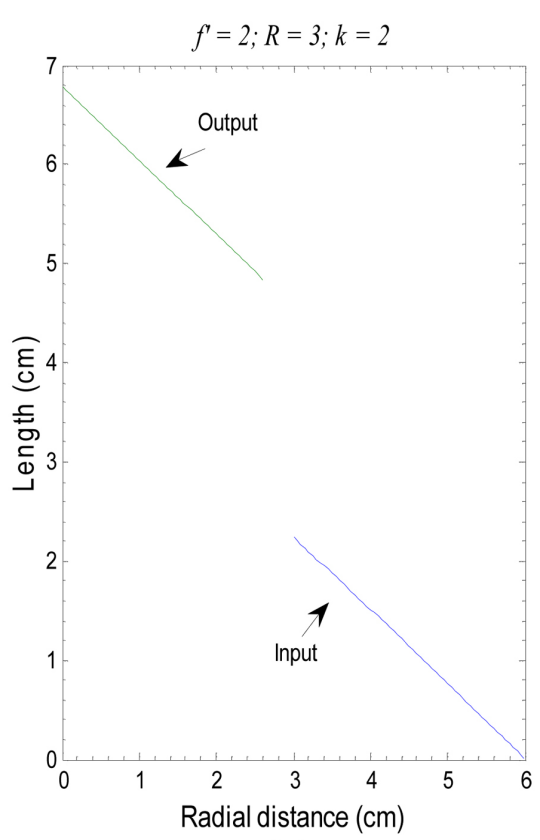

(c)

Figure 4. Effects of the inner radius $R$ on the designed surfaces for $f^{\prime}=5.75 \mathrm{~cm}, k=2$ : (a) $R=10 \mathrm{~cm}$; (b) $R=3 \mathrm{~cm}$; (c) a shorter lens system design for $f^{\prime}=2 \mathrm{~cm}, k=2, R=3 \mathrm{~cm}$. 
$R_{o}$ is the reflectivity of a central mirror in the resonator that generates the annular-Gaussian beam [2]. Solving the integral in Equation (11) leads to:

$$
\begin{aligned}
r_{o}= & \left\{\frac{R_{0} / 2}{\left(1 / w_{o}^{2}+1 / w^{2}\right)}\left[\mathrm{e}^{-2 r_{i}^{2}\left(1 / w_{o}^{2}+1 / w^{2}\right)}-\mathrm{e}^{-2 R^{2}\left(1 / w_{o}^{2}+1 / w^{2}\right)}\right]\right. \\
& \left.-w^{2}\left(\mathrm{e}^{-2 r_{i}^{2} / w^{2}}-\mathrm{e}^{-2 R^{2} / w^{2}}\right) / 2\right\}^{1 / 2} / k
\end{aligned}
$$

Note that when $M=\sqrt{2}$, then $w=w_{o}$. Consequently, Equation (12) is simplified to:

$$
r_{o}=\left(\frac{w_{o}}{k}\right) \sqrt{R_{0}\left(\mathrm{e}^{-4 r_{i}^{2} / w_{o}^{2}}-\mathrm{e}^{-4 R^{2} / w_{o}^{2}}\right) / 4-\left(\mathrm{e}^{-2 r_{i}^{2} / w_{o}^{2}}-\mathrm{e}^{-2 R^{2} / w_{o}^{2}}\right) / 2}
$$

Note that in Equation (13), the constant $k$ decreases as the radial position $r_{i}$ of the annular-Gaussian beam increases. Therefore, the input surface needs to be designed so that it attenuates the intense region of the annular-Gaussian beam while, at the same time, it redistributes the excess energy of the intense regions to the less intense peripheral region of the beam. Consequently, the surface of the first aspheric lens directs the incident rays so that they are uniformly distributed at the surface of the second aspheric lens, which then redirects the rays to be collimated.

It is worth discussing, for annular beam, the effect of the starting refracting angle $\theta_{r i}=\sin ^{-1}\left(n \sin \theta_{i i}\right)$ at the inner radius $R$ on the design. When $R$ increases, it is expected that the length $D$ will increase too. This is demonstrated in Figure 5, where $D$ is plotted versus the refracting angle for $R=1 \mathrm{~cm}$ and $R=6 \mathrm{~cm}$, for the

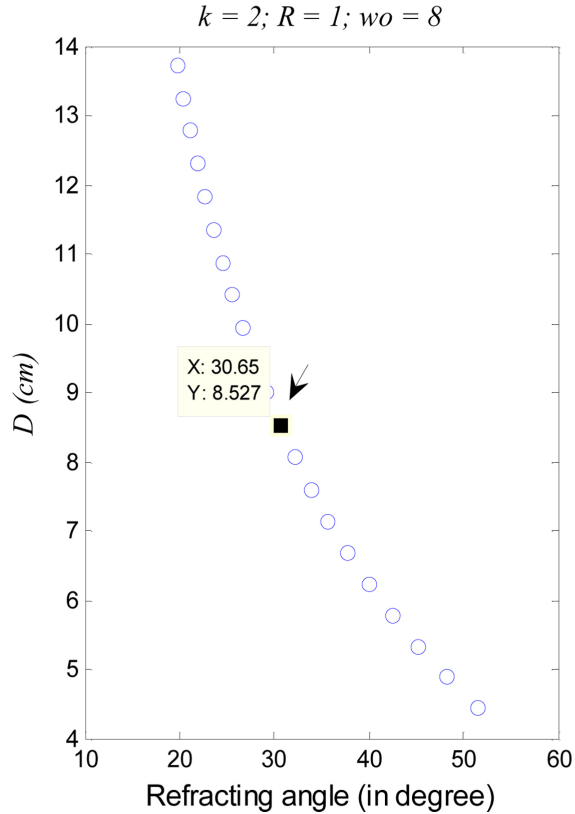

(a)

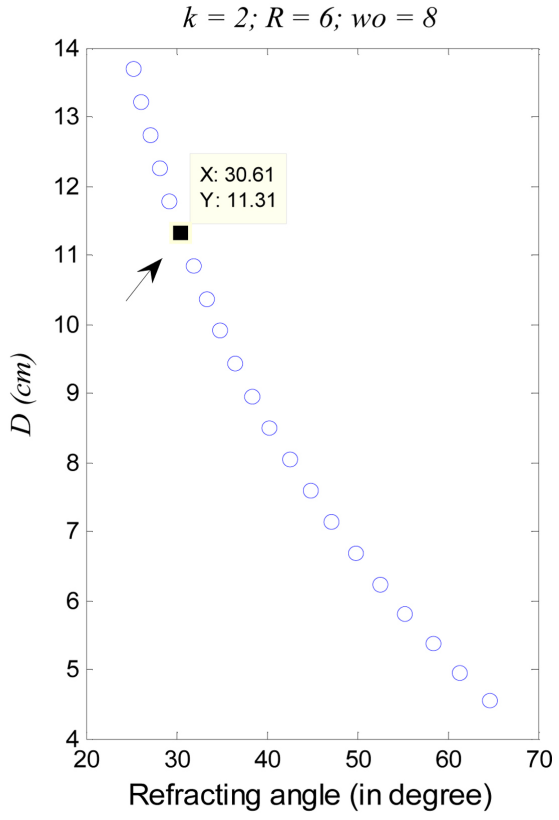

(b)

Figure 5. Effects of the inner radius $R$ of an annular beam on the starting refracting angle when $k=2, w_{o}=8$ : (a) $R=1 \mathrm{~cm}$; (b) $R=6 \mathrm{~cm}$. 


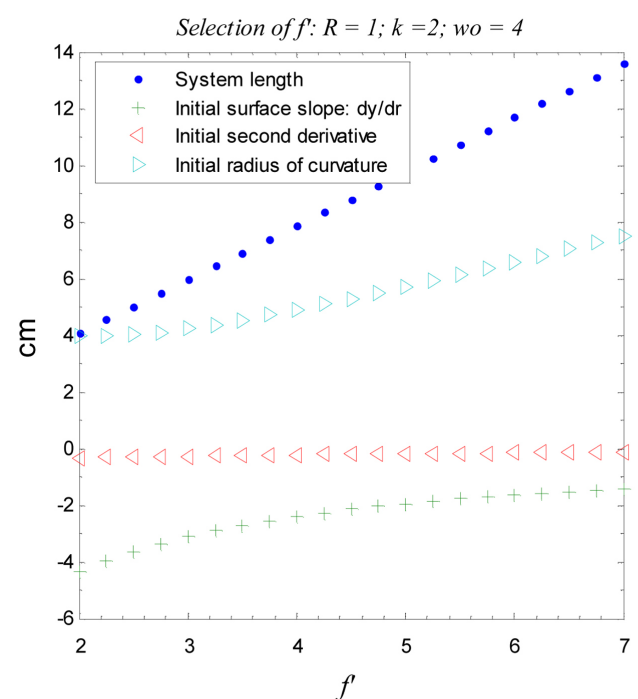

(a)

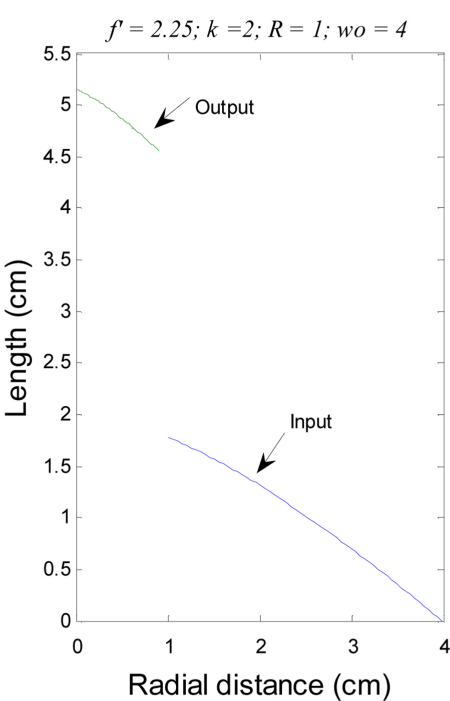

(b)

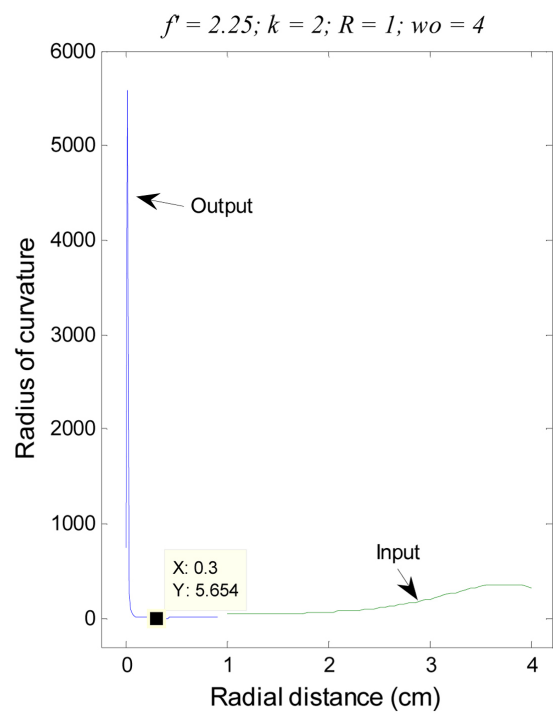

(c)

Figure 6. Two-element refracting system transformation design for annular-Gaussian to uniform with $k=2, R=1 \mathrm{~cm}, w_{o}=4:$ (a) selecting $f$; (b) the two surfaces of the lenses; (c) input and output radii of curvatures. 


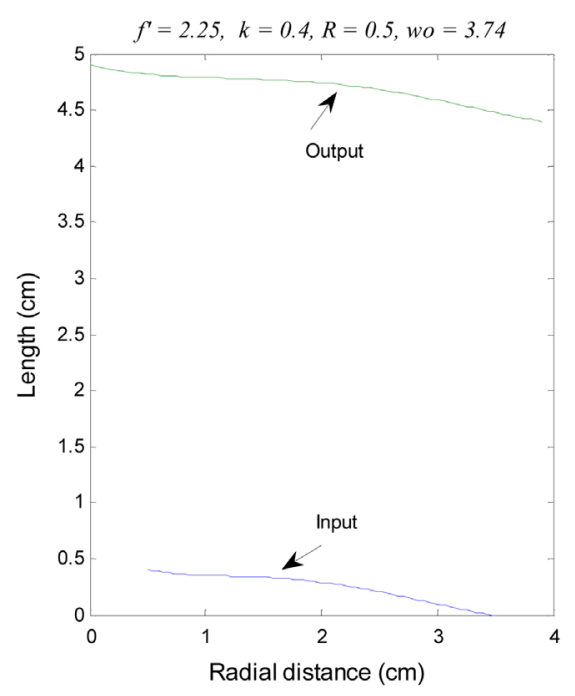

(a)

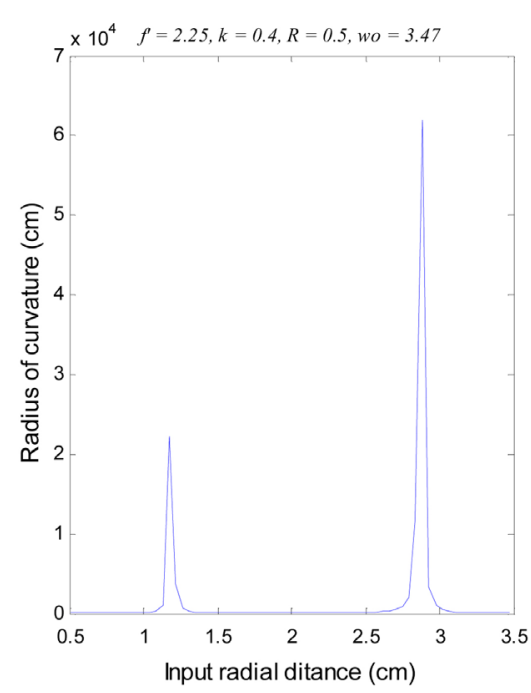

(b)

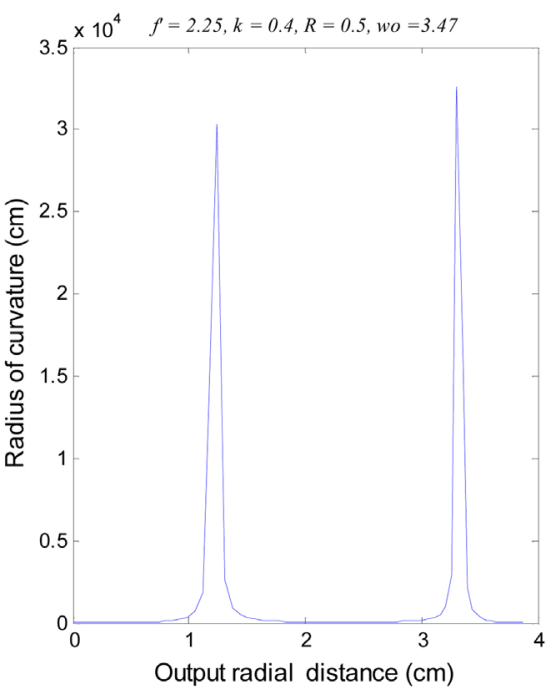

(c)

Figure 7. Two-element refracting system transformation design for annular-Gaussian to uniform with $k=0.4, R=0.5 \mathrm{~cm}, w_{o}=$ 3.74: (a) the two surfaces of the lenses; (b) input radius of curvature; (c) output radius of curvature.

radius of curvature of the output surface changes from a minimum of 9.793 to a maximum value of $3.237 \times 10^{4} \mathrm{~cm}$. These values far outperform the ones in Reference [20]. It is worth mentioning that the length of the system can be further reduced by selecting a smaller value for $f$.

Finally, the least-square curve-fitting approximate equations for the surfaces for this design are given by:

$$
\begin{aligned}
y_{i}\left(r_{i}\right)= & -(0.0038) r_{i}^{5}+(0.0592) r_{i}^{4}-(0.315) r_{i}^{3} \\
& +(0.6849) r_{i}^{2}-(0.6814) r_{i}+0.6087 \\
y_{o}\left(r_{o}\right)= & \left(9.1227 \times 10^{-4}\right) r_{o}^{5}-\left(7.6221 \times 10^{-5}\right) r_{o}^{4}-(0.0498) r_{o}^{3} \\
& +(0.1657) r_{o}^{2}-(0.2237) r_{o}+0.4931
\end{aligned}
$$

\subsection{Gaussian to Uniform Beam Transformation}

In this sub-section, we have provided designs procedure to transform the irradiance distribution of a Gaussian beam into a circularly-uniform beam using split two-element refracting system. Note that the optical set-up of Figure 1 needs to be modified to accommodate the design of the Gaussian beam profile input as illustrated in Figure 8. The power ratio between the two different beams is:

$$
k^{2}=\frac{2 \pi I_{o} \int_{0}^{r_{i}} r \mathrm{e}^{-r^{2} / w_{o}^{2}} \mathrm{~d} r}{\pi I_{o} r_{o}^{2}}=\frac{w_{o}^{2}\left(1-\mathrm{e}^{-r_{i}^{2} / w_{o}^{2}}\right)}{r_{o}^{2}}
$$

where $r_{o}$ is the radius of the circularly-uniform output beam; $I_{o}$ is the input beam peak intensity; and $w_{o}$ is the Gaussian beam radius, $r_{i}$ is the radial position of the Gaussian beam. From Equation (16), the relationships between the input and output radii are: 


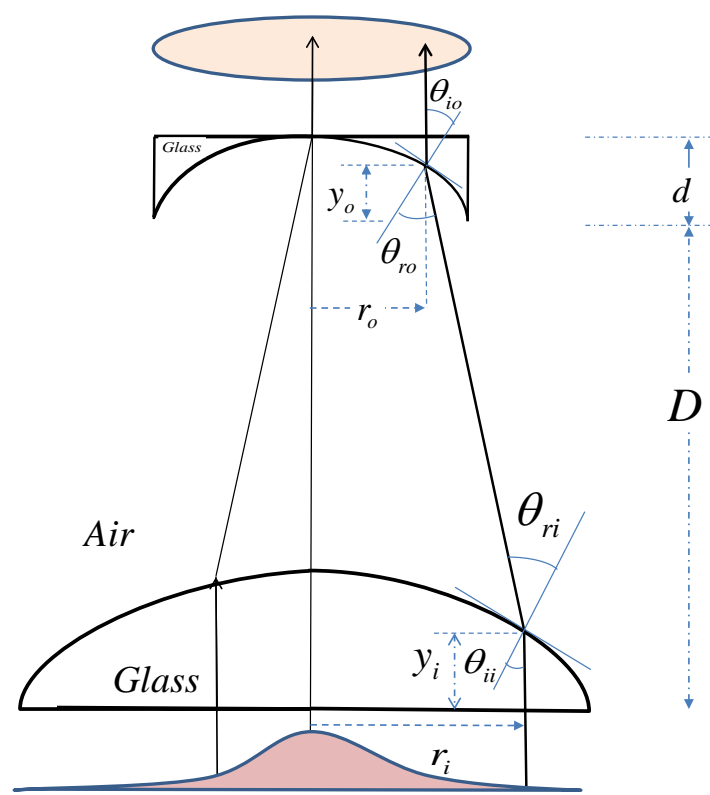

Figure 8. The optical set-up to transform Gaussian beam to uniform beam.

$$
\begin{aligned}
& r_{o}=\left(w_{o} / k\right) \sqrt{1-\mathrm{e}^{-r_{i}^{2} / w_{o}^{2}}} \\
& r_{i}=w_{o} \sqrt{-\ln \left(1-k^{2} r_{o}^{2} / w_{o}^{2}\right)}
\end{aligned}
$$

Equation (17) relates the constant $k$ to the input and output radial positions $r_{i}$ and $r_{o}$ of the lens surfaces. The beam transformation process can be explained as follows. The power in the input Gaussian beam varies inversely as the radial distance $r_{i}$. This power needs to be uniformly distributed as an output beam that has a particular radius $r_{o}$ which is determined by a constant $k$. This constant, in turn, is related to the output power concentration. As a result, every input Gaussian ray, located at a radial position $r_{p}$, will be redirected to a radial position $r_{o}$, after propagating in air such that Equation (16) holds true, i.e. $k$ remains constant.

The proposed two-element design shown in Figure 9, uses $r_{i}=2.97 \mathrm{~cm}, r_{o}=$ $1.92 \mathrm{~cm}, k=1.23, w_{o}=3$, which are the same values utilized in the one-element design in Reference [20]. However, $f$ is selected equal to $0.75 \mathrm{~cm}$ to reduce the overall system length to $2.134 \mathrm{~cm}$. For this design, the radius of curvature for input surface spans a minimum value of $12.76 \mathrm{~cm}$ to a maximum value of 255 $\mathrm{cm}$; while the radius of curvature for output surface spans a range of a minimum value of $6.392 \mathrm{~cm}$ to a maximum value of $19.83 \mathrm{~cm}$. Comparing with the one-element design of Reference [20], our proposed design is much better. The surfaces equations of the input and output lenses are given by:

$$
\begin{aligned}
y_{i}\left(r_{i}\right)= & \left(2.6314 \times 10^{-4}\right) r_{i}^{5}+(0.0032) r_{i}^{4}-(0.0198) r_{i}^{3} \\
& -(0.1099) r_{i}^{2}-(0.003) r_{i}+1.2085 \\
y_{o}\left(r_{o}\right)= & (0.0084) r_{o}^{5}-(0.0307) r_{o}^{4}+(0.0149) r_{o}^{3} \\
& -(0.1586) r_{o}^{2}+\left(4.3318 \times 10^{-4}\right) r_{o}+0.6842
\end{aligned}
$$


On the other hand, Figure 10 is a design that transforms a Gaussian beam-with a small cross-sectional area-into a circularly-uniform beam with a larger cross-sectional area. This can be achieved when the power ratio $k$ is less than 1 . The overall length system for this design is $2.64 \mathrm{~cm}$ and the approximate equations for surfaces are:

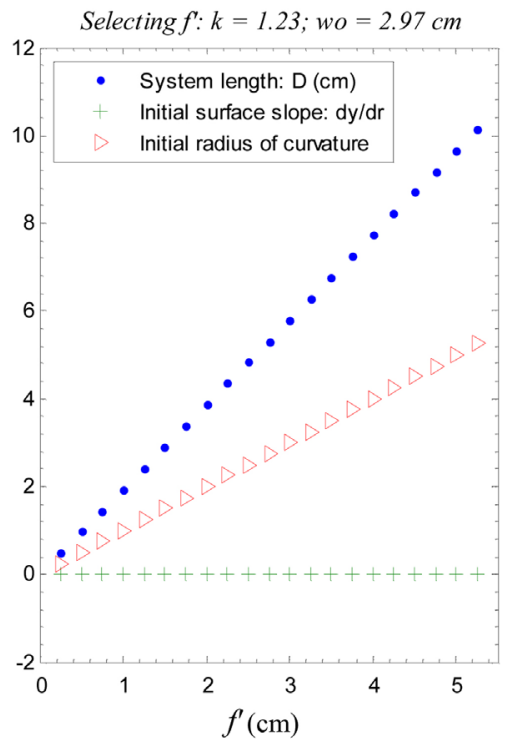

(a)

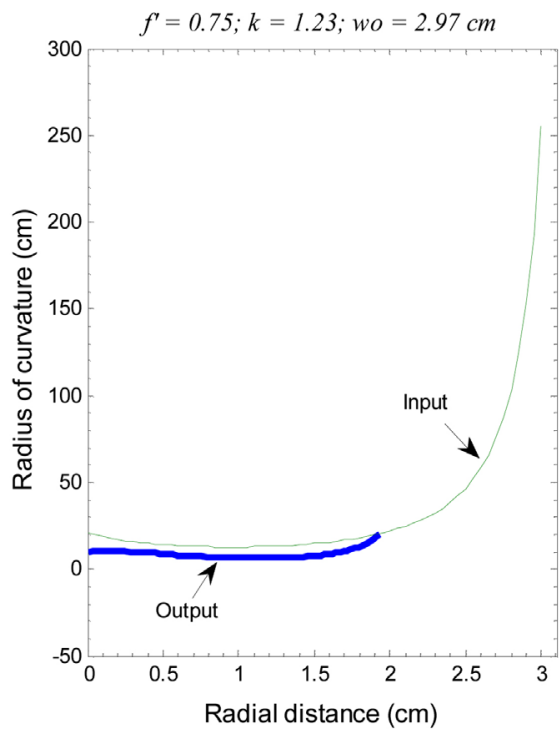

(b)

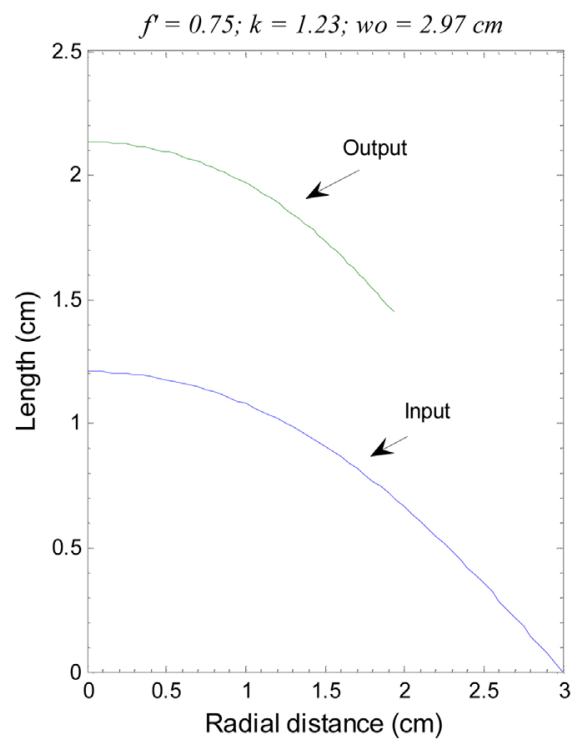

(c)

Figure 9. Two-element refracting system transformation design for Gaussian to uniform with $k=1.23, w_{o}=3$ : (a) selecting $f$; (b) the input and output radii of curvatures; (c) the two surfaces of the lenses.

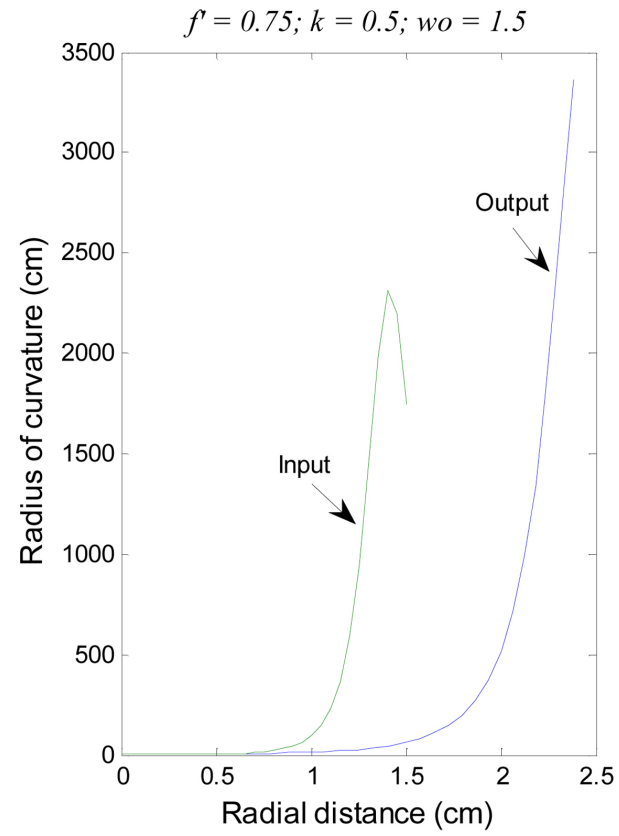

(a)

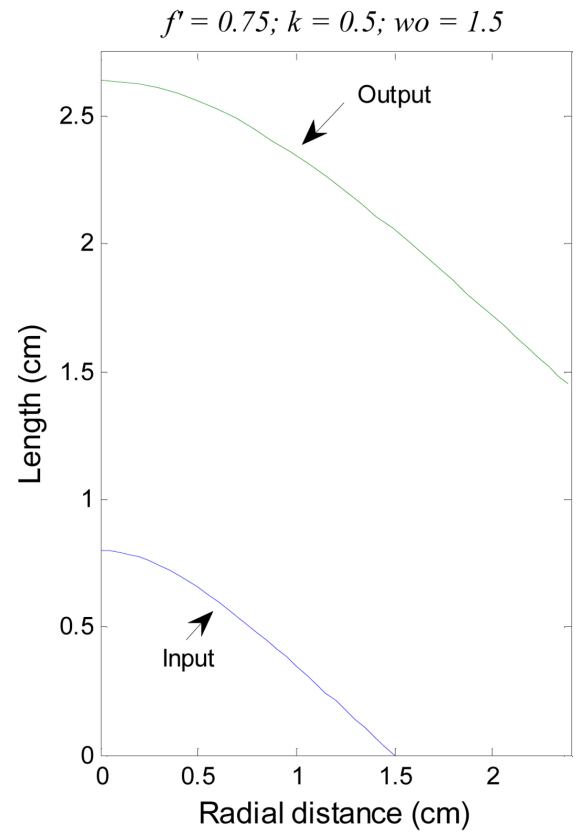

(b)

Figure 10. Two-element refracting system transformation design for Gaussian to uniform with $f^{\prime}=0.75, k=0.5, w_{o}=1.5$ : (a) the input and output radii of curvatures; (b) the two surfaces of the lenses. 


$$
\begin{aligned}
y_{i}\left(r_{i}\right)= & (0.0061) r_{i}^{5}-(0.0918) r_{i}^{4}+(0.3970) r_{i}^{3} \\
& -(0.7693) r_{i}^{2}+(0.0066) r_{i}+0.7995 \\
y_{o}\left(r_{o}\right)= & -\left(9.9011 \times 10^{-4}\right) r_{o}^{5}-(0.0014) r_{o}^{4}+(0.0756) r_{o}^{3} \\
& -(0.3695) r_{o}^{2}+(0.004) r_{o}+1.1898
\end{aligned}
$$

\section{Conclusion}

We have presented various split two-element refracting systems designs to transform three types of laser beam irradiance distributions, namely: 1) annular-uniform-to-uniform, 2) annular-Gaussian-to-uniform, and 3) Gaussianto-uniform. A detailed step-by-step design procedure is outlined. For each type of beams, we carried out rigorous analysis demonstrating the effects on the design when changing few system parameters such as the system length, the starting cone angle for annular beams, and the power ratio. In addition, the proposed two-element designs were compared to previously reported one-element designs. It was demonstrated that the proposed two-element designs are much better than the reported ones.

\section{Acknowledgements}

The authors would like to thank the reviewers for their constructive comments and suggestions that greatly improve this manuscript.

\section{References}

[1] Wilson, J. and Hawkes, J.F.B. (1987) Laser Principles and Applications. Prentice Hall International, Hertfordshire, UK.

[2] Siegman, A.E. (1986) Lasers. University Sciences Books, Mill Valley, CA, Chapter $22,23$.

[3] Ion, J.C. (2005) Laser Processing of Engineering Materials: Principles, Procedure, and Industrial Application. Elsevier Butterworth-Heinemann, Burlington.

[4] Narendra, S.P.H. and Dahotre, B. (Eds.) (2008) Laser Fabrication and Machining of Materials. Springer, New York.

[5] Sugioka, K., Meunier, M. and Pique, A. (Eds.) (2010) Laser Precision Microfabrication. Springer-Verlag, Berlin.

[6] Dickey, F.M. and Holswade, S.C. (2000) Laser Beam Shaping: Theory and Technique. Marcel Dekker, New York, 163-231. https://doi.org/10.1201/9780824741631

[7] Duocastella, M. and Arnold, C.B. (2012) Bessel and Annular Beams for Materials Processing. Laser \& Photonics Reviews, 6, 607-621. https://doi.org/10.1002/lpor.201100031

[8] Allmen, M. and Blatter, A. (1995) Laser-Beam Interactions with Materials. Springer-Verlag, Berlin. https://doi.org/10.1007/978-3-642-57813-7

[9] Duerr, F. and Thienpont, H. (2014) Refractive Laser Beam Shaping by Means of a Functional Differential Equation Based Design Approach. Optics Express, 22, 8001-8011. https://doi.org/10.1364/OE.22.008001

[10] Shealy, D. L. and Chao, S. (2003) Geometric Optics-Based Design of Laser Beam Shapers. Optical Engineering, 42, 3123-3138. https://doi.org/10.1117/1.1617311 
[11] Durnin, J., Meceli, J.J. and Eberly, H.J. (1987) Diffraction-Free Beams. Physical Review Letters, 58, 1499-1501. https://doi.org/10.1103/PhysRevLett.58.1499

[12] Cox, J. and Dibble, D.C. (1991). Holographic Reproduction of a Diffraction-Free Beam. Applied Optics, 30, 1330-1332. https://doi.org/10.1364/AO.30.001330

[13] Ma, H., Zhou, P., Wang, X., Ma, Y., Xi, F., Xu, X. and Liu, Z. (2010) Near-DiffractionLimited Annular Flattop Beam Shaping with Dual Phase Only Liquid Crystal Spatial Light Modulators. Optics Express, 18, 8251-8260. https://doi.org/10.1364/OE.18.008251

[14] Liang, J. Kohn Jr., R.N., Becker, M.F. and Heinzen, D.J. (2009) 1.5\% Root-MeanSquare Flat-Intensity Laser Beam Formed Using a Binary-Amplitude Spatial Light Modulator. Applied Optics, 48, 1955-1962. https://doi.org/10.1364/AO.48.001955

[15] Erdei, G., Szarvas, G., Lorincz, E. and Varkonyi, S. (1997) Single-Element Refractive Optical Device for Laser Beam Profiling. Proceedings of SPIE, 3100, 400-412. https://doi.org/10.1117/12.287740

[16] Hoffnagle, J.A. and Jefferson, C.M. (2000) Design and Performance of a Refractive Optical System That Converts a Gaussian to a Flattop Beam. Applied Optics, 39, 5488-5499. https://doi.org/10.1364/AO.39.005488

[17] Angelis, M., Cacciapuoti, L., Pierattini, G. and Tino, G.M. (2003) Axially Symmetric Hollow Beams Using Refractive Conical Lenses. Optics and Lasers in Engineering, 39, 283-291. https://doi.org/10.1016/S0143-8166(01)00117-8

[18] Hoffnagle, J.A. and Jefferson, C.M. (2003) Beam Shaping with a Plano-Aspheric Lens Pair. Optical Engineering, 42, 3090-3099. https://doi.org/10.1117/1.1613957

[19] Karim, M.A., Cherri, A.K., Awwal, A.A.S. and Basit, A. (1987) Refracting System for Annular Laser Beam Transformation. Applied Optics, 26, 2446-2449. https://doi.org/10.1364/AO.26.002446

[20] Jahan, S.R. and Karim, M.A. (1989) Refracting Systems for Gaussian-to-Uniform Beam Transformations. Optics \& Laser Technology, 21, 27-30. https://doi.org/10.1016/0030-3992(89)90008-X

[21] Turunen, J., Vasara, A. and Friberg, A.T. (1988) Holographic Generation of Diffraction-Free Beams. Applied Optics, 27, 3959-3962.

https://doi.org/10.1364/AO.27.003959

[22] Thewes, K., Karim, M.A. and Awwal, A.A.S. (1991) Diffraction-Free Beam Generation Using Refracting Systems. Optics \& Laser Technology, 23, 105-108. https://doi.org/10.1016/0030-3992(91)90021-F

[23] Arif, M., Hossain, M.M., Awwal, A.A.S. and Islam, M.N. (1998) Refracting System for Annular Gaussian-to-Bessel Beam Transformation. Applied Optics, 37, 649-652. https://doi.org/10.1364/AO.37.000649

[24] Iftekharuddin, K.M., Awwal, A.A.S. and Karim, M.A. (1993) Gaussian-to-Bessel Beam Transformation Using a Split Refracting System. Applied Optics, 32, 2252-2256. https://doi.org/10.1364/AO.32.002252

[25] Arif, M., Hossain, M.M., Awwal, A.A.S. and Islam, M.N. (1998) Two-Element Refracting System for Annular Gaussian-to-Bessel Beam Transformation. Applied Optics, 37, 4206-4279. https://doi.org/10.1364/AO.37.004206 